\title{
Emerging roles for telomerase in regulating cell differentiation and survival: a neuroscientist's perspective
}

\author{
Mark P. Mattson a,b,*, Weiming Fu ${ }^{\text {a }}$, Peisu Zhang a \\ ${ }^{a}$ Laboratory of Neurosciences-4F02, National Institute on Aging Gerontology Research Center, \\ 5600 Nathan Shock Drive, Baltimore, MD 21224, USA \\ ${ }^{\mathrm{b}}$ Department of Neuroscience, Johns Hopkins University School of Medicine, 725 N. Wolfe Street, \\ Baltimore, MD 21205, USA
}

Received 22 November 2000; accepted 22 November 2000

\begin{abstract}
Telomerase is a reverse transcriptase that adds repeats of a DNA sequence (TTAGGG) to the ends of chromosomes (telomeres) in mitotic cells, thus maintaining their length and preventing cell cycle arrest and cell death (cellular senescence). During development of the nervous system, telomerase activity levels are high in neural progenitor cells, but then they decrease as cells differentiate or die. The catalytic subunit of telomerase (TERT) remains at relatively high levels during the process of neuronal differentiation and then decreases sharply during the period when synapses form and programmed cell death occurs. TERT promotes survival of developing brain neurons. Suppression of telomerase activity and TERT expression promotes apoptosis of neurons, whereas overexpression of TERT prevents apoptosis by suppressing cell death at a premitochondrial step in the death cascade TERT may suppress DNA damage and/or apoptotic signals activated by damaged DNA. Recent studies of the transcriptional regulation of the TERT gene suggest that this enzyme may mediate the cell survival-promoting actions of diverse signals including estrogen, cytokines and neurotrophic factors. The elucidation of the functions of telomerase activity and TERT in neuronal differentiation and survival may lead to novel approaches for preventing neuronal death and promoting recovery of function in various neurodegenerative conditions. (C) 2001 Published by Elsevier Science Ireland Ltd.
\end{abstract}

Keywords: Alzheimer's disease; Cancer; Differentiation; Mitochondria; Neurons

* Corresponding author. Tel.: + 1-410-5588463; fax: + 1-410-5588465.

E-mail address: mattsonm@grc.nia.nih.gov (M.P. Mattson). 


\section{From cancer to brain development: roles for telomerase in cell proliferation and death}

Several articles (Rhyu, 1995; Liu, 1999) including the article by Klapper in this issue of Aging Research Reviews (Klapper et al., 2001a), have reviewed the cellular and molecular biology of telomeres and telomerase. In contrast to studies of tumor cells and proliferative somatic tissues, very few studies have examined the roles of telomerase in the nervous system, despite the fact that telomerase activity and TERT expression are quite high during development of the brain (Greenberg et al., 1998; Fu et al., 2000). Telomerase activity requires an RNA template called telomerase RNA(TR) and a catalytic protein called telomerase reverse transcriptase (TERT) (Fig. 1) Several additional telomere-associated proteins have been identified that may modify telomerase activity or otherwise affect telomere stability. (Table 1). These include: telomerase repeat-binding factor 1 (TRF1) which may inhibit telomerase activity and promote telomere shortening, TRF-2 which may stabilize telomeres, and TEP-1 which may regulate telomerase activity by interacting directly with TERT (Broccoli et al., 1997; Harrington et al., 1997; Van Seensel and de Lange, 1997). Proteins involved in DNA repair such as PARP and Ku80 might also play a role in telomere maintenance and function, and may thereby modulate cell proliferation and survival (Evans et al., 1998; d'Adda di Fagagna et al., 1999).

In somatic cells telomerase activity and TERT levels decrease during growth arrest and cellular senescence (Greenberg et al., 1998; Kim et al., 1994), and overexpression of TERT in such cells can extend their lifespan (Bodnar et al., 1998;

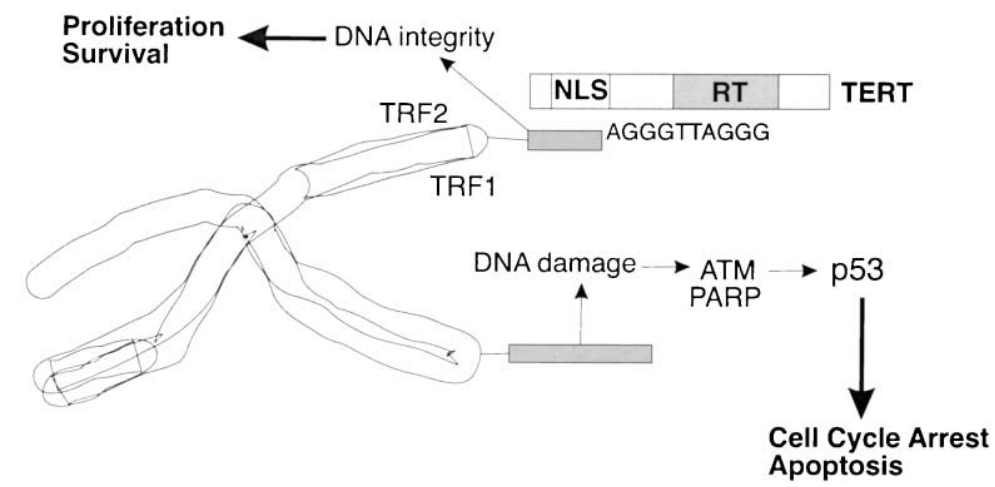

Fig. 1. Mechanisms by which telomerase may regulate cell proliferation, differentiation and survival. Telomerase activity requires an RNA component (hTR) and the catalytic reverse transcriptase protein called TERT. Telomerase prevents chromosome shortening by adding the six-base DNA repeat (TTAGGG) onto chromosome ends. DNA damage and/or chromosome shortening can activate a cell death pathway involving the ATM kinase, poly (ADP-ribose) polymerase (PARP) and the tumor suppressor protein p53. p53 promotes cell cycle arrest and apoptosis. In the nucleus TERT may suppress DNA damage-induced apoptotic signals. In the cytoplasm, TERT may suppress external apoptotic signals such as those linked to Bax translocation and caspase activation. TERT might also enhance activation of survival signal pathways, such as those activated by growth factors. NLS, nuclear localization signal; RT, reverse transcriptase. 
Table 1

Examples of telomere-associated proteins and their proposed functions

\begin{tabular}{|c|c|}
\hline Protein & Established or proposed function \\
\hline TERT & $\begin{array}{l}\text { Telomerase reverse transcriptase; telomere length maintenance, cell } \\
\text { proliferation, differentiation, survival }\end{array}$ \\
\hline TR & Telomerase RNA component; template for synthesis of TTAGGG repeats \\
\hline TRF1 & Binds telomeres; may inhibit telomerase and promote telomere shortening \\
\hline TRF2 & Telomere maintenance \\
\hline RAP1 & Telomere maintenance \\
\hline TEP1 & Binds TERT and may modulate its enzymatic activity \\
\hline Tankyrase & Poly (ADP ribose) polymerase that can ribosylate TRF1 \\
\hline PARP & Mediator of DNA damage-induced apoptosis \\
\hline Ku 80 & Telomere-associated DNA repair protein \\
\hline Dyskerin & Associates with TR; facilitates telomerase activity \\
\hline HSP90 & Chaperone that binds TERT and facilitates telomerase activity \\
\hline P23 & Chaperone that binds TERT and facilitates telomerase activity \\
\hline $\mathrm{L} 22$ & Associated with TR; function not established \\
\hline Hstau & Associated with TR; function not established \\
\hline $14-3-3$ & Binds TERT; may function in retention of TERT in the nucleus \\
\hline
\end{tabular}

Zhu et al., 1999). That telomerase can promote cell immortality is further suggested by the fact that telomerase activity is very high in most tumor cells (Kim et al., 1994), and by studies showing that organisms that undergo continuous growth with negligible aging exhibit telomerase activity in all of their somatic cells throughout life (Klapper et al., 1998a,b). Cancers can result from uncontrolled cell proliferation and/or from suppression of apoptosis (Hanahan and Weinberg, 2000). Pharmacological inhibition of telomerase or treatment with antisense oligonucleotides can inhibit growth and survival of cancer cells (Kondo et al., 1998a; Fu et al., 1999; Kondo et al., 2000), suggesting a central role for telomerase in the immortal phenotype. In the case of neural cells it has been shown that telomerase activity decreases in cells as they differentiate into neurons, and that this is associated with increased vulnerability of the cells to apoptosis (Fu et al., 1999, 2000).

Within the past 3 years, studies have shown that telomerase activity and TERT are present in the brain during embryonic development (Greenberg et al., 1998; Fu et al., 2000). When taken together with the observation that telomerase activity decreases in pheochromocytoma cells when they are induced to differentiate into a neuron-like phenotype in response to exposure to nerve growth factor (Fu et al., 1999; Kondo et al., 1998b, 2000; Kruk et al., 1996), a role for telomerase in brain development was suggested. TERT and telomerase activity are widely expressed in neuronal precursor cells during embryonic development in rats and mice (Fu et al., 2000). A detailed study of the expression of TERT, TRF1, TRF2 and telomerase activity in three different brain regions and the eye of mice at developmental stages ranging from embryonic day 13 (E13) to the adult revealed striking changes in telomerase activity and TERT expression (Klapper et al., 2001b). Telomerase activity was very high throughout the brain and in the eye at E13, then declined 
markedly between E13 and E18, and remained at a moderate level until postnatal day 3 (P3) and thereafter declined to undetectable levels by P10 (Fig. 2). Surprisingly, the sharp decrease in telomerase activity in the brain during embryonic development was not paralleled by a decrease in levels of TERT mRNA. The changes in telomerase activity and TERT expression during brain development are intriguing in that they are correlated with major developmental events including cell proliferation, differentiation and cell death. Thus, telomerase activity decreases as proliferation of neuroblasts decreases, which is also a time window when death of many newly generated neural cells may occur (Gilmore et al., 2000). On the other

(A)
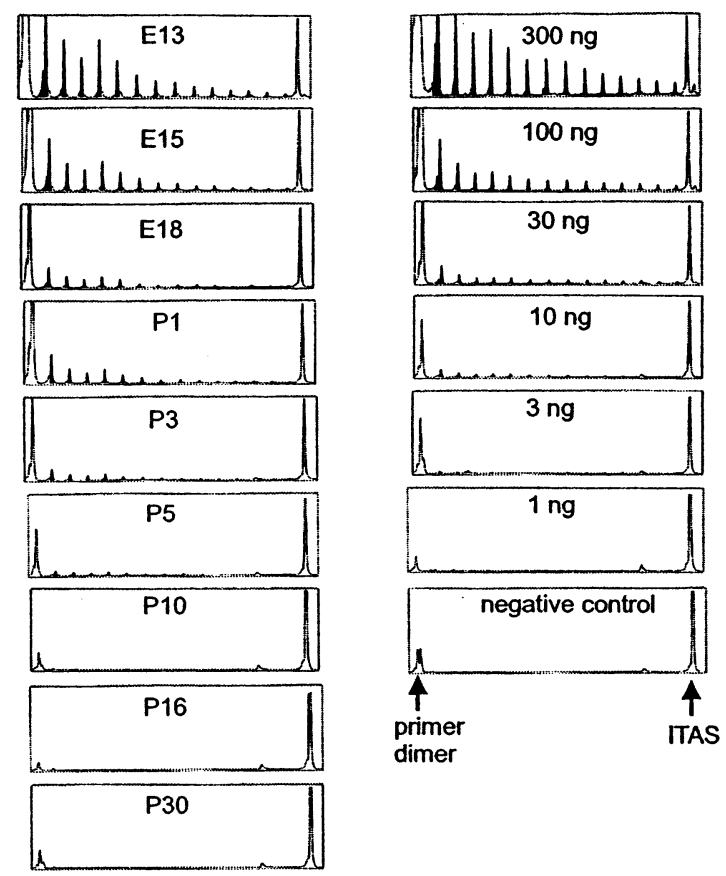

dimer

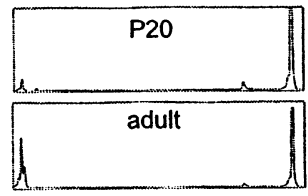

Fig. 2. Changes in telomerase activity and TERT mRNA levels in three brain regions and the eye during development suggest roles for TERT beyond telomere maintenance. (A) Relative levels of telomerase activity (determined by TRAP assay) were measured in cortical tissue from mice of the indicated ages (left column). The column at the right shows results of a serial dilution of samples from HeLa cells. The peaks between the primer dimer peak and the internal trap amplification standard (ITAS) represent telomerase activity. (B) Levels of TERT mRNA (determined by RT-PCR analysis) were measured in cortical, hippocampal, brainstem and eye tissue from mice of the indicated ages. E, embryonic day; P, postnatal day. Note that whereas telomerase activity declines sharply during embryonic development, TERT expression remains high well into the postnatal period. 

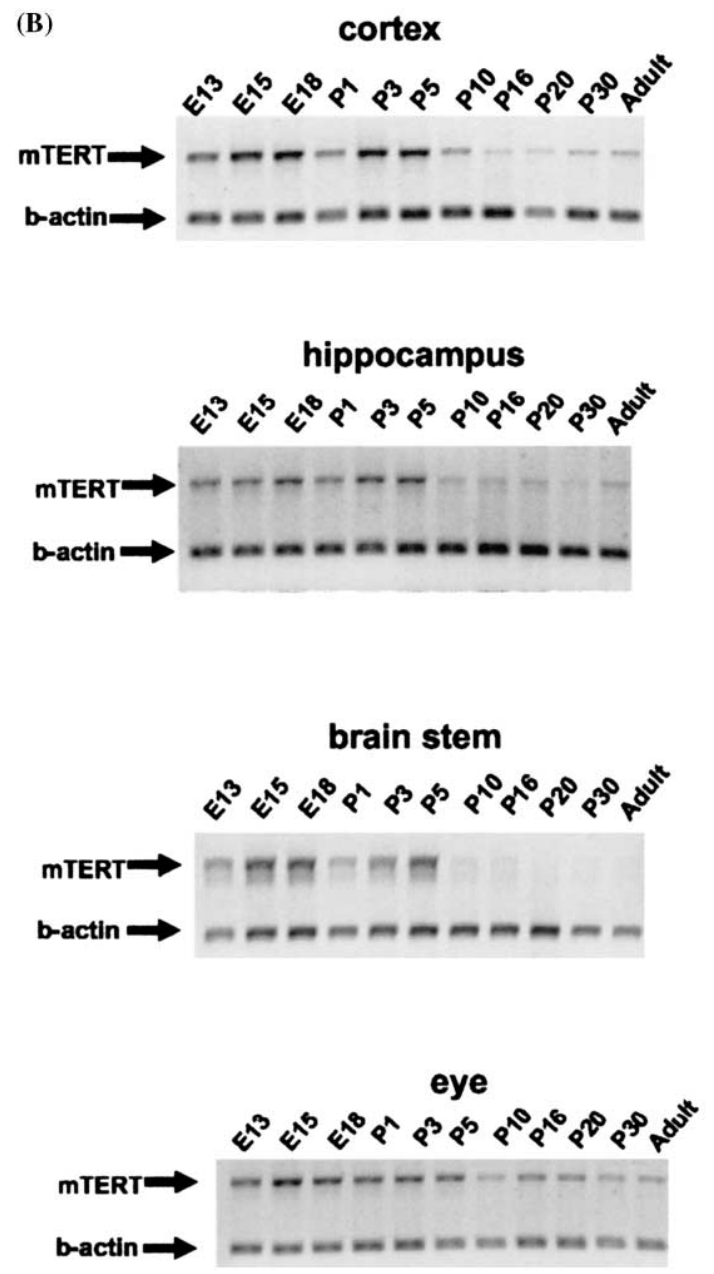

Fig. 2. (Continued)

hand, TERT levels decrease at a later stage of development in association with neuronal differentiation and synaptogenesis, a time when considerable programmed death of neurons also occurs. The dissociation between TERT levels and telomerase activity suggests that the enzyme activity is important for telomere maintenance in proliferating cells, while TERT may promote cell survival by a mechanism other than telomere maintenance. Consistent with an anti-apoptotic function of TERT in differentiated neurons are data showing that suppression of TERT expression in postmitotic embryonic hippocampal neurons can induce them to undergo apoptosis (Fu et al., 2000). The level of mRNAs encoding TRF1 and TRF2 were essentially unchanged from E13 through adulthood (Klapper et al., 2001b), suggesting that these two proteins may not play active roles in controlling brain development (although permissive roles cannot be ruled out). 
Although differentiated neurons and glial cells in the adult brain may not possess telomerase activity and express little or no TERT, there exist small populations of so-called stem cells (which are concentrated in the subventricular zone and dentate gyrus of the hippocampus) that are capable of dividing and differentiating into neurons or glial cells (Gage, 2000). Such neural stem cells taken from the adult rodent brain possess telomerase activity (Fig. 3) that can be maintained with continued propogation in cell culture (Ostenfeld et al., 2000). Neural progenitor cells in primary cultures from embryonic mice express high levels of telomerase, which rapidly decreases upon their differentiation into neurons or glia (Haik et al., 2000). Growth factors and the growth substrate may provide signals that regulate telomerase activity and/or TERT expression. As evidence, basic fibroblast growth factor causes an increase in telomerase activity and proliferation of the neural stem cells without affecting TERT levels (Haik et al., 2000). Upregulation of telomerase by neurotrophic factors might mediate the neuron survival-promoting actions of such growth factors during brain development (Mattson and Lindvall, 1997), although this remains to be established. The possibility that neurotrophic factors can induce expression of TERT and/or telmoerase activity in differentiated neurons and glia in the adult brain is currently being examined.

Interestingly, telomerase activity can be induced in cells in the adult brain in response to injury. Whereas telomerase activity was not detected in tissue samples from the hippocampus and cerebral cortex of rats under basal conditions, it was present in tissue samples from the same brain regions of rats that had been administered a seizure-inducing excitotoxin called kainic acid (W. Fu, unpublished

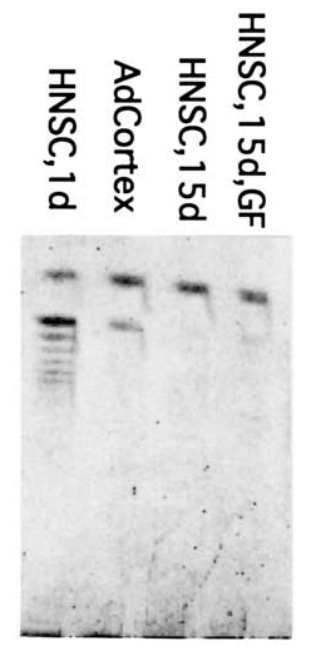

Fig. 3. Telomerase activity in neural stem cells. A standard TRAP assay was performed on homogenates of fetal human neural stem cells (HNSC) after 1 day or 15 days in culture, and on a sample from adult human cerebral cortex. Note that telomerase activity was detectable in the HNSC at 1 day in culture, but not in the HNSC that had been maintained in the absence or presence of growth factors (GF; bFGF and EGF). Telomerase activity was not detected in adult human cortical tissue. 
data). The cell type(s) in which telomerase activity appears in response to brain injury is not yet known, and the molecular mechanism responsible remains to be determined. Nevertheless, these findings suggest a role for telomerase in cellular responses to brain injury.

\section{Possible mechanisms whereby telomerase regulates cell proliferation and survival}

The current view of the mechanism by which temolerase promotes cell proliferation is based upon the concept of a cell cycle checkpoint, a point where the cell must 'decide' whether to divide or exit the cell cycle (Coffman and Studzinski, 1999). Several different proteins have been identified that play pivotal roles in the decision made at the checkpoint. These include members of the cyclin family of mitotic regulators, the ATM kinase, and the tumor suppressor protein p53. It is quite clear that shortening of telomeres beyond a critical threshold length can trigger cell cycle arrest and, accordingly, telomerase maintains cells in the proliferative state by preventing telomere shortening. Although the mechanisms that control proliferation and cell cycle exit in neural precursor cells have not been studied with the same rigor as other cell types, it is likely that many of the same molecular mechanisms apply (Learish et al., 2000).

The mechanisms whereby telomerase activity and TERT prevent cell death likely overlap with the mechanisms that control cell proliferation. Notably, signals involved in 'sensing' chromosome instability and DNA damage are likely involved in both actions of telomerase. Apoptosis is characterized by distinctive morphological features (cell shrinkage, plasma membrane blebbing and nuclear chromatin condensation and DNA fragmentation) and biochemical cascades involving proteins of the Bcl-2 and caspase families (Mattson, 2000). Overexpression of Bcl-2 results in increased telomerase activity in cancer cells (Fu et al., 1999; Mandel and Kumar, 1997), whereas overexpresion of the tumor suppressor protein PTEN decreases telomerase activity and increases spontaneous apoptosis (Tian et al., 1999). Of interest with respect to telomerase are the proteins p53, c-Myc and NF-кB (Levine, 1997; Bouchard et al., 1998; Mattson et al., 2000), P53 is a protein that responds to DNA damage by activating the apoptotic machinery, whereas c-Myc and NF- $\mathrm{BB}$ prevent apoptosis. Suppression of TRF2 function can induce apoptosis in some cell types, but not in cells that are either deficient in p53 function or lack an apoptotic response to DNA damage (Karlseder et al., 1999). Activation of the ATM kinase, which acts upstream of p53, may be necessary for telomere dysfunction-induced apoptosis and may also play a central role in the apoptotic pathway activated by DNA damage (Pandita and Dhar, 2000). TERT expression is induced by the transcription factor $\mathrm{c}-\mathrm{Myc}$, which is of considerable interest in understanding the anti-apoptotic mechanism of telomerase, because c-Myc is associated with cell immortalization and cancer (Fuhrmann et al., 1999). The upregulation of TERT by c-Myc may therefore provide an explanation for why cancer cells expressing very high levels of c-Myc do not die. Finally, the TERT gene can be induced by NF- $\mathrm{B}$ (Yin et al., 2000), suggesting a possible role for TERT

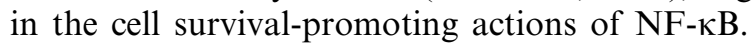


Suppresion of TERT expression in cultured embryonic brain neurons using antisense technology, causes the neurons to undergo apoptosis ( $\mathrm{Fu}$ et al., 2000), demonstrating a requirement for TERT in survival of these embryonic neurons. One mechanism whereby TERT might promote cell survival is by suppressing DNA damage, or signals generated in response to DNA damage, by stabilizing chromosome ends (Holt et al., 1999). DNA damage is well-established as a trigger for apoptosis, and telomerase can protect cells against apoptosis induced by agents known to induce DNA damage including oxidative insults and amyloid $\beta$-peptide (Fu et al., 2000; Zhu et al., 2000). The functional relationships of telomerase with DNA damage response pathways involving the ATM kinase, p53, Ku proteins and PARP (see section on cancer research above) are consistent with telomere shortening being 'sensed' as a type of DNA damage. Such DNA damage can either provide a trigger for apoptosis or can be repaired (by telomerase-mediated capping of telomeres) thus preventing apoptosis.

The possibility that TERT and/or telomerase activity mediate survival-promoting actions of well-established signals such as neurotrophic factors merits consideration in light of evidence showing that neurotrophic factors such as FGF can induce TERT expression (Haik et al., 2000). Another example comes from recent studies of the Akt kinase, a well-established mediator of survival signals activated by neurotrophic factors and other stimuli in neurons. It was recently reported that Akt phosphorylates TERT and enhances its enzymatic activity (Kang et al., 1999), suggesting a role for telomerase in the cell survival-promoting actions of Akt.

While suppression of a DNA damage response is an attractive hypothesis for the anti-apoptotic action of telomerase activity, accumulating data suggest that TERT may prevent apoptosis independently of its reverse transcriptase activity. Recent findings support a mechanism in which TERT interrupts the cell death process at a premitochondrial step (Fu et al., 2000; Zhu et al., 2000). The increased vulnerability to apoptosis of embryonic neurons with low levels of TERT is associated with mitochondrial dysfunction and caspase activation (Fu et al., 2000; Zhu et al., 2000), and treatment of TERT-deficient neurons with caspase inhibitors or cyclosporin A, an agent that blocks the mitochondrial permeability transition, prevents cell death (M.P. Mattson, unpublished data). Conversely, overexpression of TERT in pheochromocytoma cells prevents mitochondrial dysfunction and caspase activation after exposure of the cells to apoptotic insults (Fu et al., 2000). Although TERT is often localized primarily in the nucleus, in many cells including embryonic neurons it is also present in the cytoplasm (Fu et al., 2000). Because TERT acts at a premitochondrial step in the apoptotic cascade, it may modify the function of cytoplasmic proteins such as Bcl-2 family members or Par-4 that are known to control apoptosis at that point in the cell death process, although this remains to be established.

Analyses of mice lacking the RNA component of telomerase (TR) are consistent with a role for telomerase in preventing apoptosis. The $\mathrm{TR}-/-$ mice can be propogated for only a limited number of generations and then show decreased viability with age, which is associated with telomere shortening, reduced proliferation of B and T lymphocytes, and atrophy of the spleen and intestines (Blasco et al., 
Table 2

Examples of transcriptional and post-transcriptional regulation of TERT

\begin{tabular}{lll}
\hline Regulator & Effect on TERT expression or function & Reference \\
\hline NF-kB & Increased transcription & Yin et al. (2000) \\
Estrogen receptor & Increased transcription & Misiti et al. (2000) \\
Myc & Increased transcription & Kyo et al. (2000) \\
Sp1 & Increased transcription & Kyo et al. (2000) \\
MZF-2 & Increased transcription & Fujimoto et al. (2000) \\
P53 & Suppresses transcription & Xu et al. (2000) \\
Mad1 & Suppresses transcription & Gunes et al. (2000) \\
Akt & Increases enzyme activity & Kang et al. (1999) \\
\hline
\end{tabular}

1997; Herrera et al., 2000). Failure to close the neural tube during development (Herrera et al., 1999) and abnormalities in liver regeneration and enhanced cirrhosis in response to chronic liver injury (Rudolph et al., 2000) provide further evidence for increased susceptibility of cells lacking TR to apoptosis. Activation of p53 occurs in association with telomere shortening and leads to growth arrest and apoptosis in TR $-/-$ cells, and deletion of p53 abolishes the pro-apoptotic effect of TR deficiency (Chin et al., 1999). Data from TERT-deficient mice suggest that TERT $-/-$ mice are similar to TR $-/-$ mice with respect to telomere maintenance (Nikaido et al., 1999).

Finally, the promoter of TERT has been cloned and putative transcription regulatory sites identified. In several cases the regulation of TERT expression by specific transcription factors has been demonstrated. Some of the transcription factors regulating TERT expression are listed in Table 2. The emerging picture is consistent with roles for TERT in regulating cell proliferation and survival. For example, TERT expression is induced by transcription factors such as NF- $\mathrm{kB}$ (Yin et al., 2000) and estrogen receptors (Misiti et al., 2000), which are known to promote survival of a variety of cells including neurons. Conversely, TERT expression is suppressed by pro-apoptotic transcription factors such as p53 (Chin et al., 1999) and Mad (Xu et al., 2000).

\section{Mechanisms of regulation of neuronal differentiation by telomerase}

The fact that telomerase activity decreases as cells differentiate suggests a role for this enzyme in the differentiation process. One possibility is that telomerase promotes cell proliferation and that when telomerase activity decreases the cell exits the cycle and activates a differentiation program. In this view, a decrease in telomerase activity plays only a permissive role in the processes of cell differentiation. Thus, a decrease in telomerase activity during brain development may signal cells to exit the cell cycle and differentiate into neurons or glial cells. Such a mechanism has been clearly established in non-neural cells, and the available data demonstrating correlations between decreasing telomerase activity and differentia- 
tion of neural cell lines (Fu et al., 1999) and primary neurons (Fu et al., 2000) support such a mechanism. However, the trigger for differentiation is not simply the shortening of telomeres because exposure of undifferentiated PC12 cells to nerve growth factor, which does not promote telomere shortening, causes a relatively rapid downregulation of telomerase activity and differentition of the cells into a neuron-like phenotype (Fu et al., 1999). Downregulation of telomerase activity by NGF suggests that telomerase is subject to regulation by specific intracellular signal transduction cascades that involve protein kinases and transcription factors (Table 2). The fact that signals that induce cell differentiation also suppress telomerase activity is consistent with the possibility that the decrease in telomerase activity is simply a consequence of the cell differentiation process and has no particular role in controlling the differentiation process. However, treatment of cells with telomerase antisense (Kondo et al., 1998b) can induce differentiation, indicating that telomerase activity may in fact exert control on the proliferation/differentiation decision.

\section{Future directions}

There are strong correlations between telomerase activity and TERT expression and the proliferation, differentiation and survival of many types of cells. The mechanisms whereby telomerase controls these processes are beginning to be understood and include actions on signaling that regulate cell cycle arrest and apoptosis. Pressing areas for further studies include: (1) identifying proteins that interact with telomeres and TERT and establishing their roles in regulating cell proliferation and survival; (2) determining the specific mechanism whereby TERT prevents apoptosis; (3) identifying signaling pathways that regulate expression of TERT, TR and telomere-associated proteins; and (4) elucidating roles for telomerase in aging and age-related disease.

Telomerase activity, as well as TERT and other telomere-associated proteins, are now recognized as targets for drug discovery with potential applications in the treatment of cancers and neurodegenerative disorders. The goal would be to inhibit telomerase in tumor cells and to increase expression of TERT and telomerase activity in neurons. Preclinical studies support such approaches. For example, compounds that inhibit telomerase can suppress tumor growth (Kyo et al., 2000; Yamaguchi et al., 1999), and a gene therapy approach involving overexpression of TERT was effective in preventing neuronal death in experimental cell culture models relevant to the pathogenesis of Alzheimer's disease and stroke ( $\mathrm{Fu}$ et al., 2000; Zhu et al., 2000). A second approach would be to identify compounds that induce expression of TERT and telomerase activity in neurons. Studies indicate that telomerase is indeed subject to regulation by environmental signals such as neurotrophic factors (Haik et al., 2000) and estrogen (Misiti et al., 2000), and such data provide a starting point for further work in this area. In any case, the revelation that telomerase can prevent cell death in various experimental paradigms places telomere-associated proteins firmly in the field of apoptosis research and opens the 
door for future studies of telomerase in the pathogenesis of the multitude of human diseases that involve aberrant regulation of cell death.

\section{References}

Blasco, M.A., Lee, H.W., Hande, M.P., Samper, E., Lansdorp, P.M., DePinho, R.A., et al., 1997. Telomere shortening and tumor formation by mouse cells lacking telomerase RNA. Cell 91, $25-34$.

Bodnar, A.G., Ouellette, M., Frolkis, M., Holt, S.E., Chiu, C.P., Morin, G.B., et al., 1998. Extension of life-span by introduction of telomerase into normal human cells. Science 279, 349-352.

Bouchard, C., Staller, P., Eilers, M., 1998. Control of cell proliferation by Myc. Trends Cell Biol. 8, 202-206.

Broccoli, D., Smogorzewska, A., Chong, L., de Lange, T., 1997. Human telomeres contain two distinct Myb-related proteins, TRF1 and TRF2. Nat. Gen. 17, 231-239.

Chin, L., Artandi, S.E., Shen, Q., Tam, A., Lee, S.L., Gottlieb, G.J., et al., 1999. p53 deficiency rescues the adverse effects of telomere loss and cooperates with telomere dysfunction to accelerate carcinogenesis. Cell 97, 527-538.

Coffman, F.D., Studzinski, G.P., 1999. Differentiation-related mechanisms which suppress DNA replication. Exp. Cell Res. 248, 58-73.

d'Adda di Fagagna, F., Hande, M.P., Tong, W.M., Lansdorp, P.M., Wang, Z.Q., Jackson, S.P., 1999. Functions of poly(ADP-ribose) polymerase in controlling telomere length and chromosomal stability. Nat. Genet. 23, 76-80.

Evans, S.K., Sistrunk, M.L., Nugent, C.I., Lundblad, V., 1998. Telomerase, Ku, and telomeric silencing in Saccharomyces cerevisiae. Chromosoma 107, 352-358.

Fu, W., Begley, J.G., Killen, M.W., Mattson, M.P., 1999. Anti-apoptotic role of telomerase in pheochromocytoma cells. J. Biol. Chem. 274, 7264-7271.

Fu, W., Killen, M., Pandita, T., Mattson, M.P., 2000. The catalytic subunit of telomerase is expressed in developing brain neurons and serves a cell survival-promoting function. J. Mol. Neurosci. 14, $3-15$.

Fuhrmann, G., Rosenberger, G., Grusch, M., Klein, N., Hofmann, J., Krupitza, G., 1999. The MYC dualism in growth and death. Mutat. Res. 437, 205-217.

Fujimoto, K., Kyo, S., Takakura, M., Kanaya, T., Kitagawa, Y., Itoh, H., Takahashi, M., Inoue, M., 2000. Identification and characterization of negative regulatory elements of the human telomerase catalytic subunit (hTERT) gene promoter: possible role of MZF-2 in transcriptional repression of hTERT. Nucleic Acids Res. 28, 2557-2562.

Gage, F.H., 2000. Mammalian neural stem cells. Science 287, 1433-1438.

Gilmore, E.C., Nowakowski, R.S., Caviness, V.S. Jr., Herrup, K., 2000. Cell birth, cell death, cell diversity and DNA breaks: how do they all fit together? Trends Neurosci. 23, 100-105.

Greenberg, R.A., Allsopp, R.C., Chin, L., Morin, R.B., DePinho, R.A., 1998. Expression of mouse telomerase reverse transcriptase during development, differentiation and proliferation. Oncogene 16, $1723-1730$.

Gunes, C., Lichtsteiner, S., Vasserot, A.P., Englert, C., 2000. Expression of the hTERT gene is regulated at the level of transcriptional initiation and repressed by Mad1. Cancer Res. 60, 2116-2121.

Haik, S., Gauthier, L.R., Granotier, C., Peyrin, J.M., Lages, C.S., Dormont, D., et al., 2000. Fibroblast growth factor 2 up regulates telomerase activity in neural precursor cells. Oncogene 19, 2957-2966.

Hanahan, D., Weinberg, R.A., 2000. The hallmarks of cancer. Cell 100, 57-70.

Harrington, L., Zhou, W., McPhail, T., Oulton, R., Yeung, D.S., Mar, V., et al., 1997. Human telomerase contains evolutionarily conserved catalytic and structural subunits. Genes Dev. 11, 3109-3115.

Herrera, E., Martinez, A.C., Blasco, M.A., 2000. Impaired germinal center reaction in mice with short telomeres. EMBO J. 19, 472-481. 
Herrera, E., Samper, E., Blasco, M.A., 1999. Telomere shortening in $\mathrm{mTR}-/-$ embryos is associated with failure to close the neural tube. EMBO J. 18, 1172-1181.

Holt, S.E., Glinsky, V.V., Ivanova, A.B., Glinsky, G.V., 1999. Resistance to apoptosis in human cells conferred by telomerase function and telomere stability. Mol. Carcinog. 25, 241-248.

Kang, S.S., Kwon, T., Kwon, D., Do, S.I., 1999. Akt protein kinase enhances human telomerase activity through phosphorylation of telomerase reverse transcriptase subunit. J. Biol. Chem. 274, 1308513090 .

Karlseder, J., Broccoli, D., Dal, Y., Hardy, S., de Lange, T., 1999. p53- and ATM-dependent apoptosis induced by telomeres lacking TRF2. Science 283, 1321-1325.

Kim, N.W., Piatyszek, M.A., Prowse, K.R., Harley, C.B., West, M.D., Ho, P.L.C., et al., 1994. Specific association of human telomerase activity with immortal cells and cancer. Science 266, 2011-2015.

Klapper, W., Parwaresch, R., Drupp, G., 2001a. Telomerase biology in human aging and aging syndromes. Mech. Aging Dev. 122, 695-712.

Klapper, W., Heidorn, K., Kuhne, K., Parwaresch, R., Krupp, G., 1998a. Telomerase activity in immortal fish. FEBS Lett. 434, 409-412.

Klapper, W., Kuhne, K., Singh, K.K., Heidorn, K., Parwaresch, R., Krupp, G., 1998b. Longevity of lobsters is linked to ubiquitous telomerase expression. FEBS Lett. 439, 143-146.

Klapper, W., Shin, T., and Mattson, M.P. 2001b. Differential regulation of telomerase activity and TERT expression during brain development in mice. J. Neurosci. Res., in press.

Kondo, Y., Kondo, S., Tanaka, Y., Haqqi, T., Barna, B.P., Cowell, J.K., 1998a. Inhibition of telomerase increases the susceptibility of human malignant glioblastoma cells to cisplatin-induced apoptosis. Oncogene 16, 2243-2248.

Kondo, S., Tanaka, Y., Kondo, Y., Hitomi, M., Barnett, G.H., Ishizaka, Y., et al., 1998b. Antisense telomerase treatment: induction of two distinct pathways, apoptosis and differentiation. FASEB J. $12,801-811$.

Kondo, Y., Koga, S., Komata, T., Kondo, S., 2000. Treatment of prostate cancer in vitro and in vivo with 2-5A-anti-telomerase RNA component. Oncogene 19, 2205-2211.

Kruk, P.A., Balajee, A.S., Rao, K.S., Bohr, V.A., 1996. Telomere reduction and telomerase inactivation during neuronal cell differentiation. Biochem. Biophys. Res. Commun. 224, 487-492.

Kyo, S., Takakura, M., Taira, T., Kanaya, T., Itoh, H., Yutsudo, M., et al., 2000. hTERT. Nucleic Acid Res. 28, 669-677.

Learish, R.D., Bruss, M.D., Haak-Frendscho, M., 2000. Inhibition of mitogen-activated protein kinase blocks proliferation of neural progenitor cells. Dev. Brain Res. 122, 97-109.

Levine, A.J., 1997. p53: the cellular gatekeeper for growth and division. Cell 88, 323-331.

Liu, J.P., 1999. Studies of the molecular mechanisms in the regulation of telomerase activity. FASEB J. 13, 2091-2104.

Mandel, M., Kumar, R., 1997. Bcl-2 modulates telomerase activity. J. Biol. Chem. 272, 14183-14187.

Mattson, M.P., Lindvall, O., 1997. Neurotrophic factor and cytokine signaling in the aging brain. In: Mattson, M.P., Geddes, J.W. (Eds.) The Aging Brain (JAI Press, Greenwich CT). Adv. Cell Aging Gerontol. 2 299-345.

Mattson, M.P., 2000. Apoptosis in neurodegenerative disorders. Nat. Rev. Mol. Cell Biol. 1, 120-129.

Mattson, M.P., Culmsee, C., Yu, Z., Camandola, S., 2000. Roles of NF- $\kappa$ B in neuronal survival and plasticity. J. Neurochem. 74, 443-456.

Misiti, S., Anni, S.N., Fontemaggi, G., Cong, Y.S., Wen, J., Hirte, H.W., et al., 2000. Induction of hTERT expression and telomerase activity by estrogens in human overy epithelium cells. Mol. Cell. Biol. 20, 3764-3771.

Nikaido, R., Haruyama, T., Watanabe, Y., Iwata, H., Iida, M., Sugimura, H., et al., 1999. Presence of telomeric G-strand tails in the telomerase catalytic subunit TERT knockout mice. Genes Cells 4, $563-572$.

Ostenfeld, T., Caldwell, M.A., Prowse, K.R., Linskens, M.H., Jauniaux, E., Svendsen, C.N., 2000. Human neural precursor cells express low levels of telomerase in vitro and show diminishing cell proliferation with extensive axonal outgrowth following transplantation. Exp. Neurol. 164, 215-226.

Pandita, T.K., Dhar, S., 2000. Influence of ATM function on interactions between telomeres and nuclear matrix. Radiat. Res. 154, 133-139. 
Rhyu, M.S., 1995. Telomeres, telomerase and immortality. J. Natl. Canc. Inst. 87, 884-894.

Rudolph, K.L., Chang, S., Millard, M., Schreiber-Agus, N., DePinho, R.A., 2000. Inhibition of experimental liver cirrhosis in mice by telomerase gene delivery. Science 287, 1253-1258.

Tian, X.X., Pang, J.C., To, S.S., Ng, H.K., 1999. Restoration of wild-type PTEN expression leads to apoptosis, induces differentiation, and reduces telomerase activity in human glioma cells. J. Neuropathol. Exp. Neurol. 58, 472-479.

Van Seensel, B., de Lange, T., 1997. Control of telomere length by the human telomeric protein TRF1. Nature 385, 740-743.

Xu, D., Wang, Q., Gruber, A., Bjorkholm, M., Chen, Z., Zaid, A., et al., 2000. Downregulation of telomerase reverse transcriptase mRNA expression by wild type p53 in human tumor cell. Oncogene $19,5123-5133$.

Yamaguchi, F., Morrison, R.S., Takahashi, H., Teramoto, A., 1999. Anti-telomerase therapy suppressed glioma proliferation. Oncol. Rep. 6, 773-776.

Yin, L., Hubbard, A.K., Giardina, C., 2000. NF-кB regulates transcription of the mouse telomerase catalytic subunit. J. Biol. Chem. 275, 36671-36685.

Zhu, H., Fu, W., Mattson, M.P., 2000. The catalytic subunit of telomerase protects neurons against amyloid $\beta$-peptide-induced apoptosis. J. Neurochem. 75, 117-124.

Zhu, J., Wang, H., Bishop, J.M., Blackburn, E.H., 1999. Telomerase extends the lifespan of virsu-transformed human cells without net telomere lengthening. Proc. Natl. Acad. Sci. USA 96, 3723-3728. 